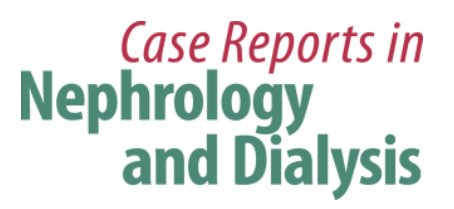

Case Rep Nephrol Dial 2018;8:56-61

DOI: $10.1159 / 000487920$

Published online: IVarch 22, 2018

\title{
Rapid and Complete Remission of Class IV Lupus Nephritis with Massive Wire Loop Lesions
}

\author{
Tomo Suzuki $^{a, b}$ Ryutaro Matsumura ${ }^{b}$ Hiroshi Kitamura $^{c}$ \\ Yugo Shibagaki ${ }^{\mathrm{a}}$ \\ aDivision of Nephrology and Hypertension, Department of Internal Medicine, St. Marianna

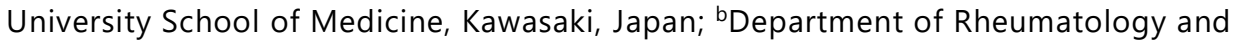 \\ Allergy, Chiba-East National Hospital, Chiba, Japan; 'Division of Immunopathology, \\ Clinical Research Center, Chiba-East National Hospital, Chiba, Japan
}

\section{Keywords}

Lupus nephritis · Wire loop lesions · Class IV-G

\begin{abstract}
Class IV lupus nephritis (LN) often has a poorer prognosis than other classes. However, class IV LN has various phenotypes, including not only segmental and global types but also others. We present the case of a 29-year-old woman with class IV-G LN who had an early response to glucocorticoid monotherapy. In addition, multiple lung nodules such as miliary tuberculosis (TB) were detected on computed tomography. All cultures of sputum, gastric fluid, and bone marrow were negative. A kidney biopsy revealed diffuse endocapillary proliferative glomerulonephritis with marked subendothelial deposition. Electron microscopy revealed massive electron-dense deposits in the subendothelial area, mesangium area, and peritubular capillaries. The histological diagnosis was class IV-G (A) LN. We administered high-dose glucocorticoid monotherapy. After treatment, the LN and the lung lesions had complete, rapid remission for 1 month. The lung lesions were associated with an immune complex similar to wire loop lesions, not TB. Thus, it is important to consider class IV-G LN with massive wire loop lesions as a new subtype.




\section{Case Reports in Nephrology and Dialysis}

\begin{tabular}{l|l}
\hline DOI: $10.1159 / 000487920$ & (c) 2018 The Author(s). Published by S. Karger AG, Basel \\
\hline
\end{tabular} www.karger.com/cnd

Suzuki et al.: Rapid and Complete Remission of Class IV Lupus Nephritis with Massive Wire Loop Lesions

\section{Introduction}

Lupus nephritis (LN) occurs in approximately $50 \%$ of patients with systemic lupus erythematosus (SLE). Patients with LN have a poorer prognosis than patients without LN, and they have an increased risk of chronic kidney disease, cardiovascular disease, and death [1]. Therefore, the pathological evaluation of $L N$ is very important.

The pathological classification of LN was revised by the International Society of $\mathrm{Ne}$ phrology/Renal Pathology Society (ISN/RPS) in 2004 [2]. We often decide the therapeutic strategy using both the American College of Rheumatology guidelines and the Kidney Disease: Improving Global Outcomes guidelines based on the ISN/RPS classification. The recommended treatment for class III and IV LN is either cyclophosphamide or mycophenolate mofetil in addition to glucocorticoids [3]. However, class III and IV LN have a variety of subtypes. Recently, it was reported that additional subtypes of LN were crescentic glomerulonephritis, tubulointerstitial lesions, renal vascular lesions, and podocyte injury [4]. However, $\mathrm{LN}$ with prominent wire loop lesions was not included as a subtype.

We herein report a case of class IV-G LN with lung involvement and massive wire loop lesions who had rapid and complete remission using glucocorticoid monotherapy.

\section{Case Report}

The patient was a 29-year-old Japanese woman. She had been experiencing a high fever for 2 weeks. In another hospital, she was diagnosed with SLE due to pancytopenia, abnormal urinalysis (proteinuria and hematuria), low complement, positive antinuclear antibody, and anti-dsDNA antibody. In addition, she had multiple small granular lesions in the lungs appearing like miliary tuberculosis (TB) on computed tomography (Fig. 1a). Therefore, she was transferred to the TB ward of our hospital. On physical examination, her height was $160.5 \mathrm{~cm}$ and her body weight was $58.8 \mathrm{~kg}$. She had a temperature of $38.8^{\circ} \mathrm{C}$. Her blood pressure was normal at $111 / 77 \mathrm{~mm} \mathrm{Hg}$. Oral ulcers and a discoid rash were observed. There was slight edema in the lower extremities. The laboratory tests showed a slightly elevated serum creatinine of $0.91 \mathrm{mg} / \mathrm{dL}$, a normal total cholesterol of $122 \mathrm{mg} / \mathrm{dL}$, a C-reactive protein of $0.57 \mathrm{mg} / \mathrm{dL}$, proteinuria of $5.1 \mathrm{~g}$ per day, and microhematuria. On serological testing, antinuclear antibody was positive, the anti-dsDNA antibody titer was very high at $400 \mathrm{IU} / \mathrm{mL}$, and serum C3 and C4 were very low at 26.4 and $4.3 \mathrm{mg} / \mathrm{dL}$, respectively. Anti-neutrophil cytoplasmic antibody was not detected. While awaiting the TB test results, she was isolated in a private room in consultation with the pulmonologist. On day 4 , her fever persisted, and laboratory tests revealed progression of pancytopenia. It was thought to be highly likely that she had hemophagocytic syndrome. Thus, we determined that this situation was emergent and initiated intravenous prednisolone (PSL) $60 \mathrm{mg}$ daily. After treatment, she immediately became afebrile. Five days after treatment, we changed from intravenous PSL $60 \mathrm{mg}$ to oral PSL $50 \mathrm{mg}$ daily. After improvement in her general condition, we performed a kidney biopsy 7 days after the start of the initial treatment. The kidney biopsy results are shown in Figure 2. Two cores of kidney tissue containing 55 glomeruli were present, none of which were globally sclerosed. Most of the glomeruli showed multiple wire loop lesions and endocapillary proliferation. The wire loop lesions were more conspicuous than endocapillary proliferation (Fig. 2a). Crescent formation, necrosis, or glomerular sclerosis were not present. In addition, vasculopathy was not present. Immunofluorescence staining demonstrated positive staining of the glomerular tuft, mesangial area, and part of the endothelial area for IgG, 


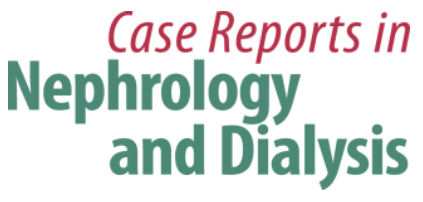

Case Rep Nephrol Dial 2018;8:56-61 DOI: $10.1159 / 000487920$

(c) 2018 The Author(s). Published by S. Karger AG, Basel www.karger.com/cnd

Suzuki et al.: Rapid and Complete Remission of Class IV Lupus Nephritis with Massive Wire Loop Lesions

IgA, IgM, C3, C1q, and fibrinogen (Fig. 2b). On electron microscopy, the glomeruli revealed massive electron-dense deposits in the endothelial and mesangial areas, but no noticeable subepithelial deposition (Fig. 2c). In addition, electron-dense deposit was present in the peritubular capillaries, but not in the tubulointerstitial area or arteries (Fig. 2d). The histological diagnosis was LN with massive wire loop lesions, ISN/RPS class IV-G (A). We did not add immunosuppressants such as cyclophosphamide or mycophenolate mofetil because we considered that LN would respond well to PSL alone. Her proteinuria and hematuria almost disappeared within 1 month. Furthermore, her lung disease disappeared after immunosuppressive therapy (Fig. 1b). In addition, we did not detect Mycobacterium tuberculosis from the sputum, gastric fluid, or bone marrow. After that, she remained in complete remission for several years using low-dose PSL only.

\section{Discussion}

The ISN/RPS classification of LN is very important when determining the appropriate therapeutic strategy to ensure kidney survival. Importantly, in the revision of the ISN/RPS classification, class IV was subdivided into segmental and global classifications. The renal prognosis of class IV is worse than that of class I, II, or III according to the ISN/RPS classification [5]. However, our case of class IV-G LN had rapid and complete remission in response to monotherapy with glucocorticoids. Class IV-G has more wire loop lesions than other classes. It is rare to observe deposition in not only in the glomeruli but also in the peritubular capillaries, as in our case. We considered that the rapid remission in our case was associated with its histological character.

Class III and IV LN have various subtypes, such as endocapillary glomerulonephritis, necrotizing crescentic glomerulonephritis associated with anti-neutrophil cytoplasmic antibody, renal vascular lesions including thrombotic microangiopathy, and focal segmental glomerulosclerosis associated with lupus podocytopathy $[4,6,7]$. On the other hand, the ISN/RPS classification distinguishes between class III and IV LN quantitatively because it considers that there is a continuum between class III and IV. Schwartz et al. [8] suggested that class III and IV represent ends of a morphologic continuum for severe LN with segmental lesions. Furthermore, they reported that severe LN had necrotizing segmental lesions and that the treatment response and prognosis were poor [9]. However, not all cases of LN progress from class III to class IV. In an experimental study, injection of IgG3-producing hybridoma led to proliferative glomerulonephritis and subendothelial depositions resembling wire loop lesions, not segmental lesions [10].

In our case, we considered that there was no transition from class III to class IV because the clinical course was drastic and segmental lesions were not identified histologically. In addition, it was recognized generally that the wire loop lesions were active lesions, but not a poor prognostic factor. In fact, the treatment response and prognosis of pure diffuse global LN with more wire loop lesions, which were without crescent or fibrinoid necrosis, were good [11]. Furthermore, Pagni et al. [12] reported that the transition rate of class IV-G to class II as mesangial proliferative glomerulonephritis at repeat biopsy was $18.9 \%$. It is unknown whether the significance of transition from class IV to class II at repeat biopsy was due to the good response to treatment or an improvement due to intensification therapy such as the combination of glucocorticoids and immunosuppressants. We considered that cases of LN with massive wire loop lesions, like our case, transitioned from class IV to class II 
after treatment as evidenced by the good response to treatment similar to mesangial proliferation in the recovery phase of acute poststreptococcal glomerulonephritis.

Class III and IV LN typically have the same treatment strategy [3]. However, they have various subtypes for a good response to initial treatment, such as that seen in our case, and a poor response such as that seen in cases of severe LN. In fact, Hanaoka et al. [13] reported recently that for complete remission at 3 years, achieving a complete renal response at 3 months was a more sensitive predictor than the use of intravenous cyclophosphamide. The response to LN treatment is associated with pathological qualities such as crescents, necrotizing lesions, and wire loop lesions. Therefore, we should consider treatment of class III and IV LN separately, taking into consideration the pathological state.

The other important concern in our case was related to the lung lesions. We suggested that the patient's lung involvement was associated with an immune complex such as wire loop lesions of LN, not TB, because her lung lesions improved after she had received immunosuppressive therapy only, and all cultures of sputum, gastric fluid, and bone marrow were negative. Chaiamnuay et al. [14] reported that SLE lung lesions with similar images revealed a nonnecrotizing granuloma of immune complex on video-assisted thoracoscopic lung biopsy. Therefore, we considered that the lung involvement was associated with an immune complex such as wire loop lesions. We suggested that the main mechanism of the kidney and lung lesions was strongly associated with the deposition of the circulating immune complex and factor.

We administered appropriate treatment with glucocorticoid monotherapy to a patient with class IV LN by considering the patient's renal pathological qualities. It is important to consider class IV-G LN with massive wire loop lesions as a new subtype.

\section{Statement of Ethics}

This article does not contain any studies with human participants or animals performed by any of the authors. Informed consent was obtained from the patient.

\section{Disclosure Statement}

The authors have no conflicts of interest or financial support to declare.

\section{References}

1 Baldwin DS, Gluck MC, Lowenstein J, Gallo GR: Lupus nephritis. Clinical course as related to morphologic forms and their transitions. Am J Med 1977;62:12-30.

2 Weening JJ, D’Agati VD, Schwartz MM, Seshan SV, Alpers CE, Appel GB, Balow JE, Bruijn JA, Cook T, Ferrario F, Fogo AB, Ginzler EM, Hebert L, Hill G, Hill P, Jennette JC, Kong NC, Lesavre P, Lockshin M, Looi LM, Makino H, Moura LA, Nagata M: The classification of glomerulonephritis in systemic lupus erythematous revisited. J Am Soc Nephrol 2004;15:241-250.

-3 Hahn BH, McMahon MA, Wilkinson A, Wallace WD, Daikh DI, Fitzgerald JD, Karpouzas GA, Merrill JT, Wallace DJ, Yazdany J, Ramsey-Goldman R, Singh K, Khalighi M, Choi SI, Gogia M, Kafaja S, Kamgar M, Lau C, Martin WJ, Parikh S, Peng J, Rastogi A, Chen W, Grossman JM; American College of Rheumatology: American College of Rheumatology guidelines for screening, treatment, and management of lupus nephritis. Arthritis Care Res (Hoboken) 2012;64:797-808.

-4 Yu F, Haas M, Glassock R, Zhao MH: Redefining lupus nephritis: clinical implications of pathophysiologic subtypes. Nat Rev Nephrol 2017;13:483-495. 


\section{Case Reports in Nephrology and Dialysis}

(c) 2018 The Author(s). Published by S. Karger AG, Basel www.karger.com/cnd

Suzuki et al.: Rapid and Complete Remission of Class IV Lupus Nephritis with Massive Wire Loop Lesions

5 Yokoyama H, Wada T, Hara A, Yamahana J, Nakaya I, Kobayashi M, Kitagawa K, Kokubo S, Iwata Y, Yoshimoto K, Shimizu K, Sakai N, Furuichi K; Kanazawa Study Group for Renal Diseases and Hypertension: The outcome and a new ISN/RPS 2003 classification of lupus nephritis in Japanese. Kidney Int 2004;66:2382-2388.

-6 Nasr SH, D'Agati VD, Park HR, Sterman PL, Goyzueta JD, Dressler RM, Hazlett SM, Pursell RN, Caputo C, Markowitz GS: Necrotizing and crescentic lupus nephritis with antineutrophil cytoplasmic antibody seropositivity. Clin J Am Soc Nephrol 2008;3:682-690.

7 Hanaoka H, Hashiguchi A, Konishi K, Kuwana M, Takeuchi T: An unusual association between focal segmental sclerosis and lupus nephritis: a distinct concept from lupus podocytopathy? CEN Case Rep 2015;4:70-75.

-8 Schwartz MM, Lan SP, Bonsib SM, Gephardt GN, Sharma HM: Clinical outcome of three discrete histologic patterns of injury in severe lupus glomerulonephritis. Am J Kidney Dis 1989;13:273-283.

-9 Behara VY, Whittier WL, Korbet SM, Schwartz MM, Martens M, Lewis EJ: Pathogenetic features of severe segmental lupus nephritis. Nephrol Dial Transplant 2010;25:153-159.

10 Itoh J, Nose M, Takahashi S, Ono M, Terasaki S, Kondoh E, Kyogoku M: Induction of different types of glomerulonephritis by monoclonal antibodies derived from an MRL/lpr lupus mouse. Am J Pathol 1993;143:1436-1443.

11 Xu S, Liu Z, Chen H, Zeng C, Zhang H, Liu Z, Hu W: Diffuse segmental and pure diffuse global proliferative glomerulonephritis: different patterns of class IV lupus nephritis. Clin Nephrol 2014;81:411-418.

12 Pagni F, Galimberti S, Goffredo P, Basciu M, Malachina S, Pilla D, Galbiati E, Ferrario F: The value of repeat biopsy in the management of lupus nephritis: an international multicentre study in a large cohort of patients. Nephrol Dial Transplant 2013;28:3014-3023.

13 Hanaoka H, Kaneko Y, Kuwana M, Takeuchi T: Early achievement of complete renal response predicts good long-term renal outcome and low systemic damage in newly diagnosed lupus nephritis class III or IV. Mod Rheumatol 2015;25:714-718.

14 Chaiamnuay S, Heck LW, Bell WC, Bastian HM: Acute granulomatous lupus pneumonitis: the first case report. Lupus 2007;16:201-204.
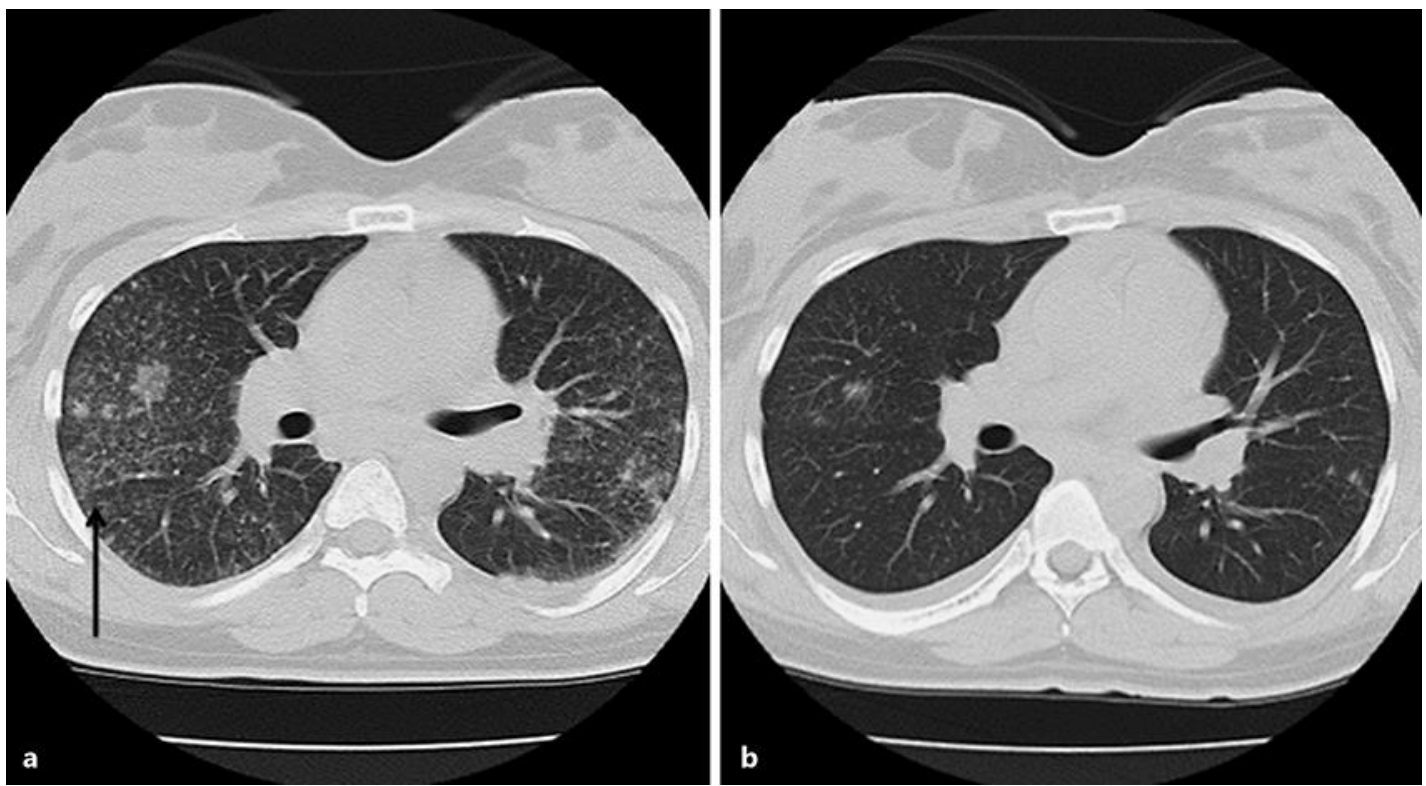

Fig. 1. Multiple small granular lesions (arrow) in the lungs on computed tomography before (a) and after treatment (b). 


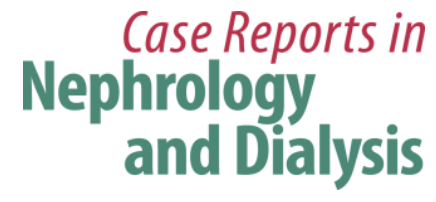 Case Rep Nephrol Dial 2018;8:56-61 DOI: $10.1159 / 000487920$
(c) 2018 The Author(s). Published by S. Karger AG, Basel www.karger.com/cnd

Suzuki et al.: Rapid and Complete Remission of Class IV Lupus Nephritis with Massive Wire Loop Lesions

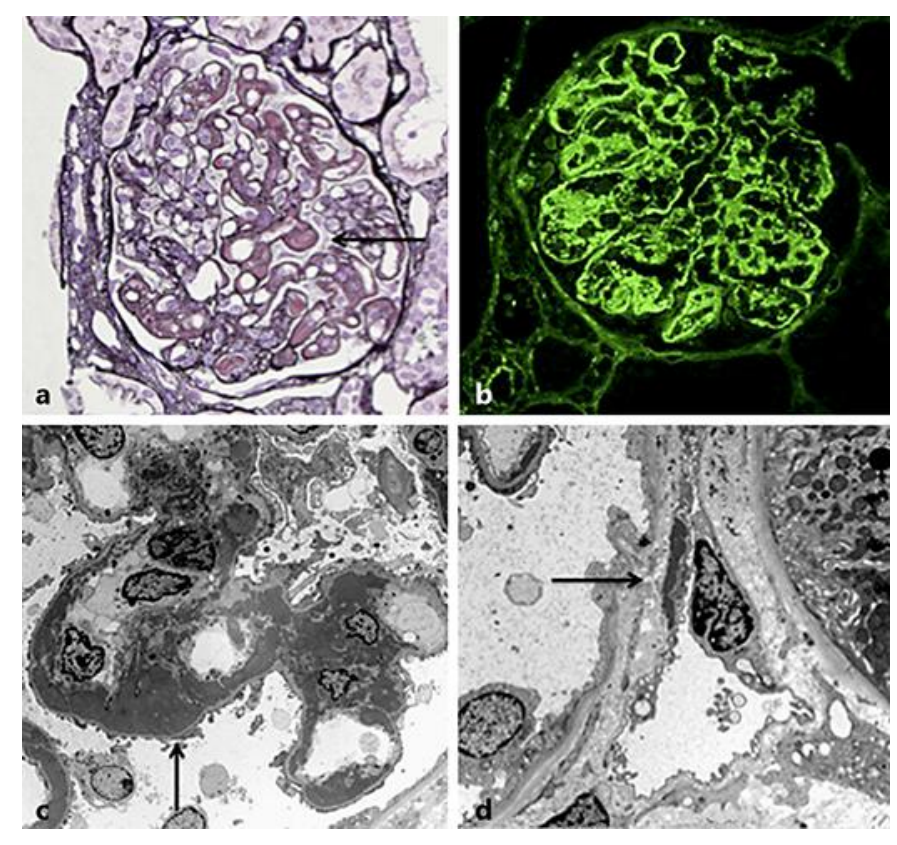

Fig. 2. Kidney biopsy specimen. a Periodic acid-Schiff-methenamine silver of the glomeruli showing massive wire loop lesions (arrow). b IgG of immunofluorescence staining showing positive staining of the glomerular tuft mesangial and partial endothelium area for IgG. c Electron microscopy of the glomeruli showing massive electron-dense deposits (arrow). $\mathbf{d}$ Electron microscopy of the peritubular capillaries showing electron-dense deposits (arrow). 